\section{Generating complex movements}

\section{R. SCHREUDER and G. GLAZENBORG Leiden University, The Netherlands}

Three problems are associated with generating complex movements on a cathode-ray tube (CRT). In the usual approach it is necessary to instruct explicitly that an object move from place $\left(\mathrm{x}_{1}, \mathrm{y}_{1}\right)$ to place $\left(\mathrm{x}_{2}, \mathrm{y}_{2}\right)$. With complex movements that have many directional changes, the number of necessary instructions can grow very rapidly.

By giving explicit instructions, it is difficult and/or time-consuming to generate complex interactions between moving objects. For instance, to give an impression of causality (cf. Michotte, 1963), it is often necessary to guarantee that the moving objects arrive at some place at exactly the same time (e.g., Object 1 touches Object 2, which then changes its direction). It is also extremely difficult to generate movements along curved paths.

To solve these problems, a subroutine package was developed that makes use of the Digital Equipment Corporation (DEC) FORTRAN graphics package, which runs on DEC VT11 and VS60 displays, operating under RSX11-M, RSX11-D, IAS, and RT-11 operating system configurations supporting FORTRAN IV.

A series of complex movements is generated in the following way: With a light pen, the user draws one or more paths on the CRT; along these paths, objects can be moved. A subroutine is supplied that can smooth these paths using a variable low-pass digital filter.

After the paths are drawn, one or more of them can be adjusted in detail, using the light pen and a pushbutton device. Furthermore, it is possible to determine the places at which the moving objects must arrive at the same time, again by using the pushbuttons. This can be done several times.

The package supplies a subroutine to generate simple polygons with variable sizes that can be used as the moving objects. Other figures can be generated by the user when the need arises.

As an example, a simple subroutine is given that can move two objects with variable speed. This subroutine is easily adjustable for moving more than two objects.

All interactions of the package with the user occur with the light pen and pushbuttons. This is extremely convenient for those cases in which the display is not near the computer.

The package consists of the following subroutines. DSPLYN draws a path on the display. DISTAN computes the distance of a path between two points. DRAW allows a tracking object to be moved with the lightpen to draw a path. STPCHA inserts steps in a given path. FILTER provides a low-pass, high cut-off filter for smoothing. ADJUST adjusts a path using the light pen. EQTIME is a routine to obtain points at which moving objects have to arrive at the same time. The velocity of the movements are accordingly adjusted. ANGLE defines a polygon with a specified number of angles and specified size. MOVE moves two objects along predefined paths with a given velocity. Three other subroutines are supplied that enable the user to supply different parameters, all using the light pen.

Availability. A listing of the package (including an example program using all the aforementioned subroutines) with more detailed instructions is available without charge from R. Schreuder, University of Leiden, - Department of Psychology, Hooigracht 15, Leiden, The Netherlands.

\section{REFERENCE}

Micнотте, A. The perception of causality. London: Methuen, 1963.

(Accepted for publication September 24, 1979.) 